\title{
Pasiyam Sa Sineng Pambansa
}

Kristian Sendon Cordero

I

Maláy man o hindi, may dalang panunukso sa akin ang pagkakahati sa siyam na kabanata (bukod sa introduksiyon) ng unang aklat ng mga kritikal na sanaysay ng eskolar na si Patrick F. Campos. Hindi maaaring maiwasang isiping tila isang sugo ng mambabarang o kontrabida ang awtor lalo na sa panahong itong madalas na ibinabandera ang sinasabing "Ikatlong ginintuang panahon ng pelikulang Filipino", at di rin malayong isiping may masamang tinapay lamang si Campos dahil sa dalang kontra-ebanghelyo ng kaniyang naturang aklat na pumapaksa sa ipinapalagay na "katapusan" o kung hindi man "kamatayan" ng tinataguriang sineng pambansa sa Filipinas. Anong tinatapos o nilalagom tungkol sa sineng Filipino ang nasa aklat ni Campos? Sa pagkakahati ng mga kabanata ng aklat ni Campos, na nagmistulang tila isang pasiyam, nais kong maghain ng ilang pagsipat sa kaniyang proyekto bilang isang manunulat at mamemelikula mula sa rehiyong Bikol. Tulad ng isang pasiyam, binaybay ko ang libro ni Campos ng may maselang pagsubaybay sa mga kabanatang nagmistulang mga bahabahagi ng isang mahabang dokumentaryo tungkol sa mga personahe at pangyayari ng ipinagbubunying ebolusyon/rebolusyon sa sineng Filipino.

Marapat na maitanong kung paanong nagkagayon na may sinasabing panibagong siglo de oro sa Filipinas samantalang kamakailan lamang ay sinabi naman ni Martin Scorsese na "Cinema is gone". Nagbago na di umano para sa direktor ang pamamaraan ng produksiyon at resepsiyon ng mga tao sa pelikula at tinukoy niyang ang pinakasalarin ay ang paglaganap ng napakaraming mga biswal at birtwal na imahen sa kasalukuyan. Napakarami na nating napapanood. Wala na tayong nakikita. Ang panonood na ito kung gayon ay dapat bang limitahan dahil ito na ang naging dahilan ng paglaho ng sine? Ani Scorsese, "Hinding-hindi na ito katulad ng kaniyang kinasayanang panonood ng sine noong araw." Para sa isang direktor na nasa loob ng malaking sarimaw pa rin, ang Hollywood, marapat lamang na timbangin ang ganitong deklarasyon. Wala na ang dating anyo ng sine ngunit gumagawa pa rin ang mga tao ng ipapakita at pagkakakitaan.

Totoong iba na rin ang moda ng panonood at maging ang mga sinehan nga sa atin katulad ng sa lungsod ng Naga ay matagal nang nagsara simula nang dumating ang cable system at lumaganap ang video rental houses, na sinundan ng naglipanang mga vcd/dvd, kasabayan ito ng paglaganap rin ng mga sex scandals mula sa mga campuses at iba't ibang sentro at bayan ng kapuluan, hanggang sa kasalukuyang panahon ng torrent, youtube atbp. Wala na ang mga antennas na noon ay inilalagay sa kawayan at unang isinisilong sa panahong may balitang paparating na bagyo. Wala na ang 
mga sinehan sa mga bayan ng Buhi, Goa, Pili, at lungsod ng Iriga. Naging mga supermarkets na ito o tindahan ng Novo at iba pang cheap goods mula Taiwan at Tsina. Ang dating Bichara 1 sa Naga ay naging repair shops na ng mga cellphones, computers, at may ilang parlors at tindahan ng pirated dvds atbp. Bagay na maaring ikakapatotoo ng sinabi ni Scorsese. Sinambit ko ito para ilarawan ang mga sapin-saping pagtingin sa sine lalo pa't may tila napakalaking tinig si Scorsese sa mundo ng pelikula. Ngunit, hindi ang sineng ito ang tinutukoy sa libro ni Campos. Bagaman makahulugan pa rin na suriin ang pahayag ng direktor, lalo pa't tila ganito rin ang nagiging taya ng pamagat ni Campos, na sa unang dampot pa lamang ay naroroon na ang paghaharubay ng isang The End, o Fin kung hindi man ng isang kamatayan, katulad ng isang takilyera noon sa Advent (isang sinehan sa Naga) na basta na lamang pinagsasaksak habang nasa loob ito ng kaniyang ticket booth. Wala na rin ang Advent sa Naga, na isa sa malalaking sinehan noong araw. Naging bangko na ito, at tindahan ng kung ano-ano, ngunit tila may sumpa raw na nakaakibat sa nasabing lugar, simula nang pinaslang ang nasabing takilyera. Wala namang kuwento-kuwento na nagmumulto ito at nangangailangan ng pasiyam. Sa personal na taya, sa Advent ko napanood ang pelikulang Kailangan Kita (2002), at sa ikalawang pagkakataon nakita ko ang Bulkang Mayon sa pinilikang tabing pagkatapos ng nauna nang pelikula ni Joel Lamangan ang Pangako ng Kahapon (1994) na may magarbong eksena ng paglilibing ng isang politiko sa simbahan ng Daraga at naroon sa frame ang pinagpipitaganang bulkan ng Bikol.

Sa Advent din nabalita na may isang kolehiyala ang naubusan ng dugo at kinalauna'y namatay dahil sa kinagat at naputol ang utong nito ng kaniyang nobyo. Sabi ng isang kaibigan, kung gusto mo rin daw na malaman kung sino ang mga patagong bakla noon sa Naga, sa Advent mo sila panakanakang makikita. Kung sasakay ng traysikel at papunta ka sa may kantong ito, maunawaan ng mga drayber kung sasabihin mong sa "dating Advent".

\section{II}

Sa pagtatalunton ko sa mga kabanata ng aklat, may mga siwang ng kaisipan at mga lundayan ng kritikal na praktika na nais kong itanghal bilang lakas, o talas ng pagsusuri ni Campos lalo pa't ang katapusang isinusulong ni Campos ay hindi ang katapusan o "end" bilang kaganapan o katuparan, kundi bilang isang pagtukoy sa delimitasyon ng espasyo, mga guwang ng kasalukuyan at namamayaning pagtanaw sa ipinapalagay na sineng pambansa, na mangyayari lamang na litisin kung hindi man tugisin ng mga panibagong metodo ng pagsipat at pagsalat sa proseso ng paglikha at pagtanggap ng isang napakaselang sining ng sine na timbangan at timbulan pa rin naman ng sosyo-politikal na kapangyarihang maaaring magsadlak sa atin sa palagiang pagtataya natin sa kung ano man ang "iginuhit $n g$ 
tadhana"1. Ibig kong sabihin, tulad ng iba pang anyo ng sining, ang sine ay maaari ring maging instrumento ng gahum na kailangan nating buwagin upang mapanatili ang kung ano mang itinataguyod nating kalayaan o pagkabuhi sa at ng sine man ito o sa panitikan. Sapagkat ang takdang ito na ginawang kasangkapan halimbawa ng mga tumakbo at nahalal na mga pangulo ng bansa, tulad nina Marcos at ng unang Macapagal, maaaring ituring na gantimpalang ipinapapala na lang sa atin ang ganoong uri ng mga pelikula na binabasbasan ng nasyonalidad o kaya'y ng etnisidad ang balintuna o kung hindi man, ang palasak na propagandistang pag-iisip na tinutukoy ng mga sanaysay ni Campos. Sa madaling salita, hindi otomatika ang nasyon. Iniluluwal ito sa pamamagitan ng pakikipagbuno. At kaya dito siya tumatawad, dito siya nanawagan ng paghunos-dili sa pamamagitan ng pagkilatis ng mga kaakibat na diskursong hindi dapat minamadali ang limi. Madaling maglagay sa kahon, mahirap ang nagpapakahon kahit sa sariling panahon. At kung nagmamadali nga naman ay parang inamin na rin naman naglilibing na nga lamang tayo. Sa karanasan ko laging huli ang nobya sa kasal kaya nahuhuli sa takdang oras ang seremonya, samantalang wala pa sa oras, o isang oras bago ang takdang misa ng patay ay dumarating na ito sa simbahan. At dahil ganap na kamatayan na nga dapat sana marahil ang tinukoy ni Campos sa usapin ng sineng pambansa, na tiyak na ikakagalit o ikakasama ng loob ng maraming tagapagtaguyod ng sineng pambansa. Isang bagay na dapat sana'y hindi natin ikinakabahala dahil hindi ba't ang kamatayan ay nagdadala din sa atin sa panibagong yugto, sa isang bagong pag-inog.

Noong taong 1999, idineklara ng manunulat na si Marne Kilates sa kauna-unahang Pagsurat Bikol sa Aquinas University sa lungsod ng Legazpi, ang di umano'y kamatayan ng kontemporanyong panitikang Bikol. May mga nagdamdam at may mga sumama ang loob sa ganoong pahayag ng tampok ng tagapanayam. May ilang pamumulsong ginawa si Kilates para ilatag ang sa tingin niyang postmortem ng nasabing panitikan halimbawa ang kawalan ng publikasyon at mga pagkilala, ang bilang ng mga manunulat, at ang mga akademyang nagtataguyod ng araling Bikol na mabibilang lang sa isang kamay ay mga indikasyon na matagal nang nalagutan ng hininga at kung gayon nasa proseso na lamang tayo ng pag-eembalsamo. Ngunit sa isang banda, ang kamatayan o katapusang iyon na ipinanukala ni Kilates ang naging mitsa para sindihan ang bangkay na iyon at mula roon, maghawan ng mga panibagong pagtalunton at landas para sa panitikang Bikol. Kung noo'y limitado lamang ang kategorisasyon na ito sa kung ano ang mga nakasulat sa Bikol-Naga o Legazpi, sa ngayon, tinutukoy at inilalathala na rin ang mga panitikang nakasulat sa wikang Bikol-Rinconada, Gubatnon, Tigaonon, Katanduanon at maging Bisakol. 
Nagsupling ng mga bagong pelikula sa wikang ito ngayong huling limang taon. Bagaman itinataguyod pa rin ang pagkilanlan sa Bikol bilang isang etnolinggwistikong grupo, hindi dapat pasubalian na may mga artikulasyong nasa loob at labas ng ethos nito, na maaari itong matapos sa panahong mag-iba ang agos ng diskurso, ang sityo ng produksyon at iba pang salik na may patungkol sa materyal na kondisyon ng paglikha ng sining. Dito ang direksyon tinatahak ko bilang isang manunulat at mamemelikula. Kung sasabihin pang bahagi ang panitikang Bikol ng panitikang pambansa, hindi dapat maging ganoon kadali ang ganoong representasyon. Kailangang mabatid na sa Bikol ay may lehiyon ng mga salita at artikulasyon at hindi dapat nagiging patabaing tupa ang rehiyon para lamang isakrispiyo sa altar ng pagsasabansa. Hindi dapat maging katapusan ang pambansa. Hindi dapat binabaog ang nasyon, sa halip, isa itong impetus na patuloy na sinasagom. Kung gayon, sa panitikan man o sa pelikula, kinakailangang maging mapagmatiyag at matiyaga pa rin sa pagkilatis sa nagpapakilalang pambansa, lalo na kung susuriin natin ang heyopolitikal na tagisan ng kapangyarihan na tumitiris din daman sa pambansa kung papalawakin na natin ang sakop ng identipikasyon ayon sa mga simtomas na binalaan na rin naman tayo. Kung kaya maging ang rehiyonal na kategorya (halimbawa, Bikol, Hiligaynon, Sebwano, Tausug) ay maaaring tukuying bernakular dahil sa labas pa rin ito ng kanon ng wikang pambansa. Ngunit kapag pinalaki mo ang sakop, nagiging rehiyonal din naman ang Filipinas sa Timog-Silangang Asya kung ilalahok na ang Indonesia, Malaysia, Thailand, Singapore atbp. Kung kaya mas madali pa ring gawin ang mga antolohiyang orihinal na nakasulat sa Ingles kaysa mga gawaing magtataguyod ng mga "rehiyonal na wika" sa mga dating mga "pambansa". Isang limitasyong hindi pa natin natatapos sapagkat nakatayo pa rin sa atin ang edipisyo ng nasyon na nagtataglay pa rin ng mga latak ng kolonyalismo hanggang sa panibagong pamamaraan ng pagdarahop dala ng sari-saring instrumento ng kapitalismo at globalismo.

Taglay ang ganitong lente, marahan kong binaybay ang aklat ni Campos sa dahilang nais ko ring siyasatin ang aking sarili lalo pa't kalahok din ako sa pagtataguyod ng isang henesis ng pagkakilanlan para sa mga Bikolnon gamit ang panitikan at ang pelikula para isulong ang mga wika at salaysay sa Kabikolan. Na maaaring sabihing bagahe o binabagahe lamang ng mga manunulat ang usapin ng pagiging Bikolnon dahil kinakailangan ko ito sa pagpapakilala ko sa sarili ko at sa aking mga likha, at sabihin pang pinapakinabangan ko rin naman kahit papano ang mga gawaing ito sa loob ng unibersidad. Samantalang sa maraming mga Bikolnon, hindi na nila kailangan pang litisin o paglimian ang kanilang mga sariling identidad dahil malinaw na itong ipinamukha o ipinatanggap sa kanila katulad ng pagiging Filipino. Gayundin naman siguro ang usapin sa sine. Manonood at manonood pa rin ang mga tao hangga't nasa sakop ito ng kanilang interes 
at maaaring maunawaan. Madaling sabihin ito ngunit hindi ako maaaring magpakulong sa ganitong argumento dahil ito rin naman ang parehong argumentong naglalayo at naghihiwalay sa atin. Gawain ng pagtuturo at pagsusulat ang pagsusuri sa suliranin, ang pagtatalab sa mga bagay na hindi lamang yaong pabalat kundi iyong humihiwa sa diwa, naglalamat sa ulirat, katulad ng sinisipat nating aklat.

\section{III}

Sa buong aklat ni Campos, walang paglalagom o hatol o parousia na nangyayari, o naganap dahil hindi naman naglatag ng isang pagbubuo o bagong balangkas ng kasaysayan ng sine sa Filipinas ang naturang proyekto. Hindi niya bagahe ang usapin ng tempus sa kaniyang diskurso ng telos. Sa katunayan, ang mismong topos at ethos ng telos ang sinisipat ni Campos kung paanong natatapos at di matapos-tapos ang pagdalumat natin sa sining ng sine bilang isang aparatus ng pagbubuntis at/o ng pagkabaog ng kung anuman ang tatawagin o itatatwa nating banwa o bayan. Dito ko kinagiliwan ang aklat sa pananalinhaga nito, sa autokritikong sipat ng pagkakasulat, kung paano ipinapaloob ng eskolar na si Campos ang kaniyang sariling tantiya at taya sa mga tekstong sangkot sa produksiyon ng sine. Walang ipinakilalang ito ang alpha at ito ang omega sa naturang aklat. Sa halip, pinagtuunan niya ng pansin ang usapin ng heyograpiya, politikal na kapangyarihan, ang global na ekonomiya at sinuri ang madali at minadaling katalogong maaaring magdala sa pagkabangkarote sapagkat mas pinipili natin ang estratehiya na walang puwang ang pagsusuri-na ang kritikal na pagsipat ay isang gawaing labag sa pagpapaunlad ng isang malaking adhikaing nakapadron sa pagpakilala sa isang uri ng sine bilang Filipino o sineng pambansa. Isang bagay na maaaring ipagkamali ng ilang mambabasa at tukuyin itong isa sa mga kahinaan ng aklat ni Campos lalo pa't laging nakakawing sa sinasabing "end" (tapos o telos?) ang pagtukoy sa isang panimula/simula.

Kung mamamarkahan ang ng pagdating ng sine sa kabisera ng sakop na kapuluan ng mga Español bilang "beginning" o simula, saan ang ipinapalagay na "end" o katapusan nito? Anong mga suliranin ang inilalatag sa atin ni Campos at ano ang mga panukala niyang pang-unawa sa mismong suliranin kaniyang hinaharaya. Dito kailangang pansining maigi ang mga pagpapaliwanag ni Campos sa kaniyang introduksiyon na kung sisipatin at kikilatisin ay maghahandog sa matalas na mambabasa ng panibagong pagdulog sa pelikulang nililikha sa kapuluang ito at kung paanong ang mismong kapuluang ito na itinuturing na isang nasyon ay nakakawing din sa iba pang mga pulo at kontinente partikular sa Estados Unidos at sa iba pang bahagi ng Timog-Silangang Asya.

Sa unang bahagi, tatlong mamemelikula ang magkakasunod niyang inihanay sa tatlong magkakasunod na kabanata upang ilatag ang mga 
sandigang kaisipan ng tungkol sa sineng ipinapakilala bilang Filipino, ang mga nilalawigan nito, ang mga direksyon ng pagkaligaw, ang likaw ng lungsod at ang lugod ng rural na espasyo at kung paano ang negosasyong ito ay pinanantili ng sine, na binubuwag din sa ibang anyo, ibang panahon, upang ipamukha sa atin na hindi kailanman naikakahon ang kabalintunaan, babala o baliktad man itong tanggapin ng mga nakatanod, ng mga nanaguyod. Sa katunayan, maaaring basahin na magkakaugnay na sanaysay ang unang tatlong kabanata tungkol sa diskurso ng lungsod sa pelikulang Manila by Night ni Bernal, ang lokasyon at posisyon ni Mike De Leon sa Philippine Cinema at si Kidlat Tahimik bilang isang native fimmaker. Dahil ginawaran ng estado si Bernal ng Pambansang Alagad ng Sining sa Pelikula, na malaon ay maaari ding mangyari sa dalawang direktor (de Leon at Tahimik), madali na lamang na ikawing ang mga pelikula at persona ng mga direktor na ito sa kung anuman ang maituturing at maitatatwa nating pambansa at di-pambansa. O malamang ay hindi rin ito maigawad sa kanila sapagkat labas ang dalawa sa esensya ng kapangyarihan ng pambansa. Saan nga ba mailalagay ang katulad ni De Leon? O si Kidlat Tahimik na maaaring nakulong na rin sa kaniyang sariling esensya. Bihag na ng sariling bahag?

Dito nagiging mas matalas sa kaniyang sipat si Campos dahil ang mismong nasyong magtataguri o hindi magtataguri ang mismong isinasalang niya sa isang problematikong espasyong dapat sana'y kinakapalooban sa tuwina ng diskurso at talinhaga, sapagkat nasa puno't dulo naman lagi ang politika at ang estetika sa panitikan man ito o sa pelikula. Pansinin ang iba't ibang tinig at estilo ang mahihinuha sa tatlong sanaysay, at mapapansing may laging bukas na lagusang itinuturo si Campos, upang maging bagong sityo ng diskurso. Dito niya tayo laging hinahamon na manahan at kung kinakailangan lumikas din upang maghanap ng mga panibagong anyo ng pagdulog sa anyo ng sine, na hindi na mababakuran basta na lamang ng diskurso ng pambansa. Maaari kayang iwaksi na ang espirito at esensya ng nasyon o ng bansa upang higit nating malinang ang mga dalan?

Sa panitikan, ang ganitong mga hangganan at limitasyon ng mga wika at kultura ay nilulusaw ng kapitag-pitagang gawain ng pagsasalin. Sa sine, ang paglikha ng pelikula ang mismong nagiging anyo ng pagluwas sa mga pagtatakda, kasabay na usapin na rin palagi rito ang komodipikasyon at distribusyon ng materyal, ang kasiningan at ang popularidad nito, ang mga isyu patungkol $\mathrm{sa} / \mathrm{ng}$ identidad at pagmamay-ari, na hindi rin naman malayo sa kung paano tinantanggap ang kung anong panitikan at hindi panitikan sa bansang ito.

Kung gayon, ang ganap na pagtanggap sa sine bilang "pambansa" na walang pagkilala sa masalimuot na naratibo ng pag-usbong ng anyong ito sa kapuluang ito, bagay na inilatag niya sa ikaapat na kabanata (Cinemalaya and the Politics of Naming a Movement), o ang hindi pagkilala sa kaugnay na negosyo (kapital) at negosasyong (kita) na nagaganap sa mismong proseso 
ng pagbubuo ng pelikula, o ang espektakulong nagmumulto sa atin bilang mga pook ng kadalisayan, sa Banaue man ito sa banwa nin mga Engkanto, o sa lalawigan ng Dalagang Bukid, o ng Huling Balyan at naimamapa lamang o natunton lamang nang gamiting "lokasyon" ang mga mga sityo ng pelikula. O kaya'y mga katawang nagbabalik-bayan o hindi na kailanman makakabalik ng bayan, sapagkat nilusaw na rin naman ng mga anyo at produksyon ng mga pelikula ang mga espasyo at lunsaran, ang mga ito'y mga patibong na naghihintay sa atin sa daan ng marami pang Kalimugtong. Sa huling kabanata, ipinakita ni Campos kung paano ang espektakulo ng pambansa ay multong nakabantay sa atin at ito ang puhunan ng mas matinding takot, sapagkat maging sa panahong ibinabandila natin ang ikatlong ginintuang panahon ng sine, nananatiling kuwento/kuwenta pa rin ang mga salaysay ng mga transnasyonal na katawan ng mga OFW, ang mga hati-hati ang katawan katulad ng buong kapuluan. Silang mga naglalaho at nagiging mga bituin sa pinilakang tabing, ang mga "lulubog-lilitaw sa saradong hukay" ng telon, at telon itong katulad ng eternal na lungsod ng maraming pagbulusok at pagkabulok, ng Maynila, ang kinakapitang kapital, sa loob o labas man ito ng ating kapuluan, ang nananatiling terra incognito.

Sa wikang Bikol, ang salita para sa panonood ay dalan. (Dalan kitang sine/Manood tayo ng sine.) Ito rin ang salita para sa daan. Sa taya ko, ang aklat ni Campos ay isang maalwan na dalan. Siksik at liglig sa mga pananalig sa mga interseksyon, sa mga pagkrus-lihis-landas ang kaniyang mga sanaysay na malamang na ikadismaya ng iba pang mambabasa na marami na rin sigurong sapantaha at alintana sa nasabing pag-aaral ng sineng tinataguriang Filipino. Maaari din namang ikapurga o kung hindi man ikabagot ng ilan ang aakalaing patalun-talon na mga argumento. Lalo pa't sa kaniyang pagtalakay ng iba pang anyo ng sine, walang pakundangang sinisipi ni Campos ang mga pelikula na parang namumulot na lamang ito ng kung anong pelikulang aakma sa kaniyang isinusulong na mga diskurso. Bagay na hindi niya pa ganap na naisakatuparan sa ilang bahagi ng libro sapagkat, ika nga niya, hindi niya naman sinimulang buuin ang mga sanaysay sa pag-aambisyong magiging isang ganap itong libro na may ganitong tila napakalaking proklamasyon. Ganun pa man, kung mamarapating pumaimbulog, may kagyat na animo'y isang mahaba-habang pelikula ang buong libro ni Campos, bagay na lubos kong ikinalugod, o kaya'y isang mahabang talaarawan ng isang nagmamahal sa sine at sa lahat ng kabulig nito at mapalad tayong binabasa natin ito, at umasa tayong marami pang siwang at rurok, liblib, mapanganib na pagdidiskurso ang gagawin ng eskolar na si Campos sa mga darating pang mga panahon. Isang pagdalan ang pagbabasa ng panganay na aklat ni Campos, panonood ito, malalim na panonood, sapagkat tanging sa ganitong masalimuot na posisyon lamang nalilikha ang mga dalan, ang mga lokasyon, ang bagong habitus. 


\section{Sanggunian}

Campos, P. F. (2016). The end of national cinema: Filipino film at the turn of the century. Diliman: University of the Philippines Press.

Kilates, M. (1999). Speech as guest speaker for the first Pagsurat Bikol, Aquinas University., Philippines.

Coyle, J. (2016, December 20). Martin Socrsese:'cinema is gone.' AP News. Retrieved from https:// apnews.com/931d13ebfb6245e0b00169d7447208d2/martin-scorsese-cinema-gone

\section{Notes}

'Noong taong 1965 inilabas ang pelikulang Iginuhit ng Tadhana: The Ferdinand Marcos Story bilang isang pamamaraan ng panibagong uri ng pangangampanya sa bansa. Sa parehong taon din ng halalan ipinalabas ang Tagumpay ng Mahirap sa direksyon nina Lamberto Avellana, Gerardo de Leon, at Eddie Romero (na pawang magiging mga Pambansang Alagad ng Sining Sa Pelikula). Ang pelikula'y tungkol naman sa buhay ni Diosdado Macapagal na tumatakbo rin sa pagkapangulo; ang naturang pelikula'y tumayong pantanpat sa pelikula tungkol kay Marcos.

KRISTIAN SENDON CORDERO is a poet, translator and filmmaker from Bikol. He wrote and directed the first film of Nora Aunor in her mother tongue, Hinulid, (2016, Quezon City International Festival) His recent collections of poetry Labi (Ateneo de Manila University Press) and Canticos: Apat na Boses (UST Publishing House) received the National Book Awards and Gintong Aklat Awards for Best Book in Philippine Literature. As a translator, he has translated the works of Rainer Maria Rilke, Jorge Luis Borges and Oscar Wilde. His first film Angustia (CinemaOne Originals, 2013) was recognized as one of the Best First Films by the Young Critics Circle of the Philippines. He teaches at Ateneo de Naga University and Mapua University (Intramuros) (corresponding author: agnuspress@gmail.com). 